\title{
ISOMORPHISM THEOREMS FOR INFINITE ORDER DIFFERENTIAL OPERATORS
}

\author{
J. D. BUCKHOLTZ
}

\begin{abstract}
The operators studied are infinite order linear differential operators with constant coefficients. We require that the characteristic function of the operator be analytic in a neighborhood of 0 and have a radius of convergence which is less than that of its reciprocal. It is shown that such operators may be regarded as isomorphisms between Banach spaces of entire functions. These Banach spaces, in every case, are isomorphic to the sequence space $c_{0}$. Further, translation in the domain plane defines an automorphism on both the domain and range space of the operator.
\end{abstract}

Let $\varphi(z)=\sum_{j=0}^{\infty} b_{j} z^{j}$ have radius of convergence $r(0<r<\infty)$ and suppose that $\theta(z)=1 / \varphi(z)=\sum_{j=0}^{\infty} a_{j} z^{j}$ has radius of convergence greater than $r$. Let $D$ denote the derivative operator and denote by $\varphi(D)$ the operator which takes an analytic function $u$ into

$$
\varphi(D) u=\sum_{j=0}^{\infty} b_{j} u^{(j)}
$$

It is natural to take for the domain of $\varphi(D)$ the family of entire functions $u$ such that (1) is uniformly convergent on every compact set [3, Lemma 1.1]. Subject to this requirement on its domain, the behavior of the operator $\varphi(D)$ can be completely determined. Under suitable norms, the domain and range of $\varphi(D)$ are Banach spaces of entire functions, and $\varphi(D)$ is an isomorphism (i.e., a linear homeomorphism) between these spaces. An explicit determination of these spaces was obtained in [1], where it was shown that the spaces are completely determined by the singularities of $\varphi$ on the circle $|z|=r$. The basic structure of the spaces is, however, independent of the function $\varphi$. In every case, they are isomorphic to $c_{0}$, the space of complex sequences with limit 0 , normed with the supremum norm. The proof given in [1] for this result relied heavily on infinite matrices and to some extent on the Appell polynomials generated by $\varphi$. The purpose of the present paper is to give a somewhat altered proof which avoids these difficulties.

Received by the editors January 18, 1973.

AMS (MOS) subject classifications (1970). Primary 34A35, 47E05; Secondary 30A98, 46E15. 
Since $\theta=1 / \varphi$ is analytic in the closed disk $|z| \leqq r$, it follows that $\varphi$ is zero-free in the closed disk $|z| \leqq r$, and that its singularities on $|z|=r$ are poles. Let $\alpha_{q}^{-1}, 1 \leqq q \leqq \lambda$, denote the (distinct) poles of $\varphi$ on $|z|=r$, and let $m(q)$ denote the order of the pole $\alpha_{q}^{-1}$. Set $m=\max m(q), 1 \leqq q \leqq \lambda$. It was shown in [3] that the domain of $\varphi(D)$ is the space $\mathscr{U}$ of entire functions $u$ such that each of the series

$$
\sum_{k=0}^{\infty}\left(\begin{array}{c}
k+m(q)-1 \\
m(q)-1
\end{array}\right) \alpha_{q}^{k} u^{(k)}(0), \quad 1 \leqq q \leqq \lambda,
$$

converges. For $u \in \mathscr{U}$ we set

$$
\|u\|_{0}=\max _{1 \leq q \leq \lambda} \sup _{0 \leq n<\infty}\left|\sum_{k=n}^{\infty}\left(\begin{array}{c}
k+m(q)-1 \\
m(q)-1
\end{array}\right) \alpha_{q}^{k} u^{(k)}(0)\right| .
$$

Let $Q(z)=\prod_{a=1}^{\lambda}\left(1-\alpha_{q} z\right)^{\min \{m(q) \cdot m-1\}}$ and let $\mathscr{F}$ denote the space of entire functions $f$ such that

$$
\lim _{n \rightarrow \infty} r^{-n}\left(D^{n} f\right)(0)=\lim _{n \rightarrow \infty} n^{m-1} r^{-n}\left(D^{n} Q(D) f\right)(0)=0 .
$$

For $f \in \mathscr{F}$, set

$$
\|f\|_{1}=\sup _{0 \leqq n<\infty}\left(\frac{1}{2}\right)\left\{r^{-n}\left|\left(D^{n} f\right)(0)\right|+\left(\begin{array}{c}
n+m-1 \\
m-1
\end{array}\right) r^{-n}\left|\left(D^{n} Q(D) f\right)(0)\right|\right\} .
$$

It was shown in [3] that $\mathscr{F}$ is the range of $\varphi(D)$. The following result is an immediate consequence of Theorem 1.5 of [3] and the Mapping Theorem of [1].

THEOREM 1. The operator $\varphi(D)$ has domain $\mathscr{U}$ and range $\mathscr{F}$. Further, $\varphi(D)$ is 1-1, bounded, and possesses a bounded inverse. Its inverse is the restriction to $\mathscr{F}$ of the operator $\theta(D)$.

The operator $\varphi(D)$ defines an isomorphism between $\mathscr{U}$ and $\mathscr{F}$, and this is the principal tool needed to show that $\mathscr{F}$ is isomorphic to $c_{0}$. Let $E_{r}$ denote the space of all entire $f$ such that $f^{(n)}(0)=o\left(r^{n}\right), n \rightarrow \infty$, and, for $f \in E_{r}$, set

$$
\|f\|_{2}=\sup _{0 \leqq n<\infty}\left|r^{-n} f^{(n)}(0)\right| .
$$

The identification of $f$ with the sequence $\left\{r^{-n} f^{(n)}(0)\right\}_{0}^{\infty}$ defines a linear isometry between $E_{r}$ and $c_{0}$; we shall use differential operators to construct an isomorphic map of $\mathscr{F}$ onto $E_{r}$.

Apart from Theorem 1, the construction depends on three observations about the spaces $\mathscr{F}$ and $\mathscr{U}$. First, note that if $m=1$ ( $\varphi$ has only simple poles on $|z|=r)$, then $Q(z) \equiv 1$ and $\mathscr{F}$ is the space $E_{r}$. The second observation is slightly deeper and crucial to the argument: the polynomial $Q$, 
and therefore the space $\mathscr{F}$, can not distinguish between a pole of $\varphi$ of order $m$ and a pole of order $m-1$. In particular, if we let

$$
v_{1}(z)=\theta(z) \prod_{m(q)=m-1}\left(1-\alpha_{q} z\right), \quad \mu_{1}(z)=\frac{1}{v_{1}(z)},
$$

and let $\mathscr{F}\left(\mu_{1}\right)$ denote the space obtained from $\mathscr{F}$ by replacing $\varphi$ by $\mu_{1}$, then $\mathscr{F}\left(\mu_{1}\right)=\mathscr{F}$. Since we shall have to consider several spaces, we shall sometimes write $\mathscr{F}(\varphi)$ and $\mathscr{U}(\varphi)$ for $\mathscr{F}$ and $\mathscr{U}$ and use $\mathscr{F}(\mu)$ and $\mathscr{U}(\mu)$ to denote the spaces obtained by replacing $\varphi$ by a function $\mu$. The norm on $\mathscr{U}(\mu)$ which corresponds to $\|\cdot\|_{0}$ will be denoted by $N_{0}(; \mu)$, and the norm on $\mathscr{F}(\mu)$ which corresponds to $\|\cdot\|_{1}$ will be denoted by $N_{1}(; \mu)$. In addition to $\mu_{1}$ and $\nu_{1}$, we shall also need the functions

$$
v_{2}(z)=\prod_{m(a) \geqq m-1}\left(1-a_{q} z\right), \quad \mu_{2}(z)=\frac{1}{v_{2}(z)}, \quad \text { and } \quad \mu_{3}(z)=\left\{\mu_{2}(z)\right\}^{m} \text {. }
$$

Our third observation concerns the space $\mathscr{U}$. It is possible to replace the norm $\|\cdot\|_{0}$ by an equivalent norm $\|\cdot\|_{3}$ which depends only on the poles of $\varphi$ of orders $m$ and $m-1$. The convergence of

$$
\sum_{k=0}^{\infty}\left(\begin{array}{c}
k+m(q)-1 \\
m(q)-1
\end{array}\right) \alpha_{q}^{k} u^{(k)}(0)
$$

for values of $q$ for which $m(q)=m$ obviously implies the convergence of (2) for values of $q$ such that $m(q) \leqq m-2$. Therefore $\mathscr{U}$ is the space of all entire $u$ such that (2) converges for all $q$ such that $m(q) \geqq m-1$. It is not hard to show that the norm

$$
\|u\|_{3}=\max _{m(q) \geqq m-1} \sup _{0 \leqq n<\infty}\left|\sum_{k=n}^{\infty}\left(\begin{array}{c}
k+m(q)-1 \\
m(q)-1
\end{array}\right) \alpha_{q}^{k} u^{(k)}(0)\right|
$$

is equivalent to the norm $\|\cdot\|_{0}$ (a proof of this is contained in [1, Lemma 4.1]). In the space $\mathscr{U}(\mu)$ we shall write $N_{3}(; \mu)$ for the norm corresponding to $\|\cdot\|_{3}$.

Let $I$ denote the identity operator and let $\Gamma_{m-1}$ denote the operator which transforms an entire function $f$ into

From the factorization

$$
\left(\Gamma_{m-1} f\right)(z)=\sum_{k=0}^{\infty}\left(\begin{array}{c}
k+m-1 \\
m-1
\end{array}\right) f^{(k)}(0) \frac{z^{k}}{k !} .
$$

$$
\Gamma_{m-1}=\frac{1}{(m-1) !} \prod_{t=1}^{m-1}(z D+t I),
$$

one sees that $\Gamma_{m-1}$ is a differential operator of order $m-1$ with polynomial coefficients. 
We are now in a position to establish an isomorphism between $\mathscr{F}$ and $E_{r}$. We have seen that $\mathscr{F}=\mathscr{F}(\varphi)$ is the same space as $\mathscr{F}\left(\mu_{1}\right)$. Theorem 1 applied to the operator $\mu_{1}(D)$ establishes an isomorphism between $\mathscr{F}\left(\mu_{1}\right)$ and $\mathscr{U}\left(\mu_{1}\right)$; specifically, $\nu_{1}(D)$ is an isomorphic mapping of $\mathscr{F}$ onto $\mathscr{U}\left(\mu_{1}\right)$.

We now show that the identity transformation defines an isomorphism between $\mathscr{U}\left(\mu_{1}\right)$ and $\mathscr{U}\left(\mu_{3}\right)$. Note that $\mu_{1}$ has no poles of order $m-1$ on $|z|=r$ and the poles of $\mu_{1}$ of order $m$ on $|z|=r$ are the poles of $\varphi$ of orders $m$ and $m-1$ on $|z|=r$. To write down the $N_{0}\left(; \mu_{1}\right)$ norm of a member of $\mathscr{U}\left(\mu_{1}\right)$ is fairly complicated unless one introduces new names for the poles of $\mu_{1}$. There is, however, no difficulty in writing down the equivalent norm $N_{3}\left(; \mu_{1}\right)$. We have, for $u \in \mathscr{U}\left(\mu_{1}\right)$,

$$
N_{3}\left(u ; \mu_{1}\right)=\max _{m(q) \geqq m-1} \sup _{0 \leqq n<\infty}\left|\sum_{k=n}^{\infty}\left(\begin{array}{c}
k+m-1 \\
m-1
\end{array}\right) \alpha_{q}^{k} u^{(k)}(0)\right| .
$$

Note that $\mathscr{U}\left(\mu_{1}\right)$ and $\mathscr{U}\left(\mu_{3}\right)$ contain the same functions and that $N_{3}\left(u ; \mu_{1}\right)=N_{0}\left(u ; \mu_{3}\right)$; this justifies our assertion about the identity map.

Let $V$ denote the image of $\mathscr{U}\left(\mu_{3}\right)$ under the operator $\Gamma_{m-1}$, and for functions $v=\Gamma_{m-1} u$ in $V$, set

$$
\begin{aligned}
\|v\|_{4} & =\left\|\Gamma_{m-1}^{\prime} u\right\|_{4}=N_{0}\left(u ; \mu_{3}\right) \\
& =\max _{m(q) \geqq m-1} \sup _{0 \leqq n<\infty}\left|\sum_{k=n}^{\infty}\left(\begin{array}{c}
k+m-1 \\
m-1
\end{array}\right) \alpha_{q}^{k} u^{(k)}(0)\right| .
\end{aligned}
$$

This choice of norm makes $\Gamma_{m-1}$ a linear isometry and extends our isomorphism from $\mathscr{F}$ to $V$. For $v=\Gamma_{m-1} u$ we have $v^{(k)}(0)=\left(\begin{array}{c}k+m-1 \\ m-1\end{array}\right) u^{(k)}(0)$, $k=0,1,2, \cdots$. Therefore

$$
\|v\|_{4}=\max _{m(q) \geqq m-1} \sup _{0 \leqq n<\infty}\left|\sum_{k=n}^{\infty} \alpha_{q}^{k} v^{(k)}(0)\right| .
$$

Note also that $v \in V$ if and only if $\sum_{k=0}^{\infty} \alpha_{q}^{k} v^{(k)}(0)$ converges for all $q$ such that $m(q) \geqq m-1$. One sees therefore that $V$ normed with $\|\cdot\|_{4}$ is the same space as $\mathscr{U}\left(\mu_{2}\right)$ with norm $N_{0}\left(; \mu_{2}\right)$.

Theorem 1 applied to $\mu_{2}(D)$ asserts that $\mu_{2}(D)$ is an isomorphic map of $V=\mathscr{U}\left(\mu_{2}\right)$ onto $\mathscr{F}\left(\mu_{2}\right)$. But the poles of $\mu_{2}$ on $|z|=r$ are all simple, so that from our first observation we obtain $\mathscr{F}\left(\mu_{2}\right)=E_{r}$. Retracing our steps, we see that the isomorphism between $\mathscr{F}$ and $E_{r}$ is established by the mappings

$$
\begin{aligned}
\mathscr{F} & =\mathscr{F}\left(\mu_{1}\right) \stackrel{\nu_{1}(D)}{\longrightarrow} \mathscr{U}\left(\mu_{1}\right) \stackrel{I}{\longrightarrow} \mathscr{U}\left(\mu_{3}\right), \\
\mathscr{U}\left(\mu_{3}\right) \stackrel{\Gamma_{m-1}}{\longrightarrow} V & =\mathscr{U}\left(\mu_{2}\right) \stackrel{\mu_{2}(D)}{\longrightarrow} \mathscr{F}\left(\mu_{2}\right)=E_{r} .
\end{aligned}
$$

This proves the following. 
THEOREM 2. The operator $\mu_{2}(D) \circ \Gamma_{m-1} \circ v_{1}(D)$ is an isomorphic map of $\mathscr{F}$ onto $E_{r}$.

For a fixed complex number $w$, let $T_{w}$ denote the operator which transforms an entire function $f$ into $\left(T_{w} f\right)(z)=f(z+w)$. It is worth noting that the spaces $\mathscr{U}$ and $\mathscr{F}$ are translation invariant, i.e., the restriction of $T_{w}$ to either space is a bijection. As one would expect, the operator $T_{w}$ (restricted to either space) is bounded and has a bounded inverse.

THEOREM 3. The operator $T_{w}$ defines an automorphism on each of the spaces $\mathscr{U}$ and $\mathscr{F}$.

Proof. The function $\mu(z)=e^{w z} \varphi(z)$ has the same singularities as $\varphi$. Therefore $\mathscr{U}(\mu)=\mathscr{U}, \mathscr{F}(\mu)=\mathscr{F}$, and $\mu(D)$ is an isomorphic map from $\mathscr{U}$ to $\mathscr{F}$. Note that $T_{w}=\exp \{w D\}$. The proof depends on showing that $\mu(D)$ has the operator factorizations

$$
\mu(D)=\varphi(D) \circ T_{w}
$$

and

$$
\mu(D)=T_{w} \circ \varphi(D) .
$$

For the time being we take these for granted. Suppose that $u \in \mathscr{U}$. From (3) we have $\mu(D) u=\varphi(D)\left\{T_{w} u\right\}$. Applying $\theta(D)$ to both sides of this equation, we obtain $T_{w} u=\theta(D)\{\mu(D) u\}$. Therefore the restriction to $\mathscr{U}$ of $T_{w}$ satisfies $T_{w}=\theta(D) \circ \mu(D)$, and it follows from Theorem 1 that this is an automorphism.

Suppose now that $f \in \mathscr{F}$, and set $u=\theta(D) f$. From (4) and Theorem 1 we obtain $\mu(D) u=T_{w}\{\varphi(D) u\}=T_{w} f$. But $\mu(D) u=\mu(D)\{\theta(D) f\}$. Therefore $T_{w} f=\mu(D)\{\theta(D) f\}$, and the restriction to $\mathscr{F}$ of $T_{w}$ satisfies $T_{w}=\mu(D) \circ$ $\theta(D)$. As before, it follows that this restriction is an automorphism.

To establish (3) and (4), we need some information about Appell polynomials. Let $\left\{p_{k}\right\}_{0}^{\infty}$ and $\left\{\rho_{k}\right\}_{0}^{\infty}$ denote the Appell polynomial sequences generated by $\varphi$ and $\mu$ respectively. It is not hard to show that $\rho_{k}(z)=$ $p_{k}(z+w)$. We shall also need the fact [3, Theorem 2.1] that, for $u \in \mathscr{I}$, the expansions

and

$$
\{\varphi(D) u\}(z)=\sum_{k=0}^{\infty} u^{(k)}(0) p_{k}(z)=\sum_{k=0}^{\infty} u^{(k)}(w) p_{k}(z-w)
$$

are valid.

$$
\{\mu(D) u\}(z)=\sum_{k=0}^{\infty} u^{(k)}(0) \rho_{k}(z)=\sum_{k=0}^{\infty} u^{(k)}(w) \rho_{k}(z-w)
$$


For $u \in \mathscr{U}$ we have

$$
\begin{aligned}
\{\mu(D) u\}(z) & =\sum_{k=0}^{\infty} u^{(k)}(0) \rho_{k}(z)=\sum_{k=0}^{\infty} u^{(k)}(0) p_{k}(z+w) \\
& =\sum_{k=0}^{\infty} u^{(k)}(w) p_{k}(z)=\left\{\varphi(D)\left(T_{w} u\right)\right\}(z) .
\end{aligned}
$$

This establishes (3). To establish (4), note that

$$
\{\mu(D) u\}(z)=\sum_{k=0}^{\infty} u^{(k)}(0) \rho_{k}(z)=\sum_{k=0}^{\infty} u^{(k)}(0) p_{k}(z+w)=\left\{T_{w}\{\varphi(D) u\}\right\}(z) .
$$

Therefore $\mu(D) u=T_{w}\{\varphi(D) u\}$, and the proof is complete.

\section{REFERENCES}

1. J. D. Buckholtz, Appell polynomial expansions and biorthogonal expansions in Banach spaces, Trans. Amer. Math. Soc. (to appear).

2. - Appell polynomials whose generating function is meromorphic on its circle of convergence, Bull. Amer. Math. Soc. 79 (1973), 469-472.

3. - Appell polynomials and differential equations of infinite order, Trans. Amer. Math. Soc. (to appear).

4. R. D. Carmichael, On nonhomogeneous linear differential equations of infinite order with constant coefficients, Amer. J. Math. 58 (1936), 473-486.

5. I. M. Sheffer, Systems of linear differential equations of infinite order, with constant coefficients, Ann. of Math. 30 (1928/29), 250-264.

Department of Mathematics, Uiniversity of Kentucky, Lexington, Kentucky 40506 